\title{
Paragonimiasis: First Case Reported in Brazil
}

\author{
Antônio Carlos Moreira Lemos ${ }^{1,2,5}$, João Carlos Coelho ${ }^{3}$, Eliana Dias Matos ${ }^{1,4}$, Guilherme Montal ${ }^{1,6}$ Fernanda Aguiar $^{1,6}$, \\ and Roberto Badaró ${ }^{2}$ \\ ${ }^{1}$ Octávio Mangabeira Hospital; ${ }^{2}$ Medicine School of Federal University of Bahia; ${ }^{3}$ Laboratory of Pathology of José Silveia Foundation; \\ ${ }^{4}$ Medicine School and Public Health of Bahia; ${ }^{5}$ Center of Pneumologist Research of Octávio Mangabeira Hospital; ${ }^{6}$ Cárdio Pulmonar Institute; \\ Salvador, BA, Brazil
}

\begin{abstract}
The authors present a case from a 59 years old white female Brazilian patient, based in Salvador-Bahia, Brazil's northeastern side area, who experienced irritative cough and progressive dyspnea, and, after 18 months, was admitted to a hospital with respiratory insufficiency. The physical exam showed diffuse rales in both hemithoraces. Initial leukogram showed 14,400 cells/ $\mu \mathrm{L}$ with $14 \%$ of eosinophils and chest $X$-ray showed peribronchovascular infiltrate, predominating in the lower half of the lung fields, and small opaque nodules. The high-resolution computed tomography scan of the chest (HRCT) presented compatible pattern with airways disease, especially from the small airways, with air trapping, tree sprouting images, central lobular nodules and bronchiectasis, making the results compatible with bronchiolitis and bronchiectasis. The transbroncho biopsy unveiled granulomatous lesion with necrosis, where was noticed a structure compatible to a parasitic case, and the research of the parasite eggs in the sputum was positive to paragonimus. After the praziquantel use, the patient presented a thick ferruginous expectoration and the result for BAAR examination was positive. The PCR exam and the sputum culture confirmed $M$. tuberculosis, and then the treatment for $M$. tuberculosis was initiated. The authors warn that this infection may have been a consequence of economics globalization process, where the importation of parasitized crustaceans might be the cause. However, there is the need of an accurate examination for the possibility of paragonimus specimens in this area of Brazil.

Key-Words: Paragonimiasis, tuberculosis, respiratory insufficiency, bronchiolitis Brazil, case report.
\end{abstract}

Paragonimiasis is the result of an infection caused by one described type of paragonimus [1]. There are 50 described species of paragonimus and 10 of them are harmful to human beings. The most common is the Paragonimus westermani, which is endemic in China, Japan, Philippines and Korea. Other variety have been responsible for the contamination of human beings on the west side of Africa, South America and Central America [2]. Infections caused by $P$. kellicotti have been autochthonous within the United States of America [3], but most of the cases had been diagnosed on immigrants. No case was reported in Brazil so far. Most of the time, the contamination happens when the raw or undercooked crustaceans (crab or crayfish) infested by paragonimus on metacercaria stage are ingested. Eggs eliminated on the sputum or on human stools, in fresh water, after a while, release miracidia, which move freely through the water and contaminate the water snails. Inside them, the miracidia develop into cercariae and, once eliminated, they penetrate on the crustacean's muscle and then unfold to metacercariae. The social habit of ingesting raw food makes the ingestion of contaminated crustaceans the main cause of this disease. Other ways of contamination are: eating raw meat of mammal animals (dog, cat), which are also paragonimus final hosts; and through

Received on 23 October 2005; revised 4 December 2006.

Address for correspondence: Dr. Antônio Carlos Moreira Lemos. Núcleo de Pesquisa em Pneumologia (NUPEP) - HEOM. Praça Conselheiro João Alfredo, S/No ${ }^{\circ}$, Pau Miúdo, Salvador - Bahia. Zip code: 40.320-350 Telephones: +55 (71) 386-4122 (Extension 233 \& 272)/ 245-1249/ 9987-9102. E-mail: acmlemos@uol.com.br.

The Brazilian Journal of Infectious Diseases 2007;11(1):153-156. (C) 2007 by The Brazilian Journal of Infectious Diseases and Contexto Publishing. All rights reserved. contaminated utensils [2]. The metacercariae, in human duodeno, are released from the cysts and penetrate into the gastrointestinal wall, moving into the peritoneal cavity. Even though there is the chance of migration to the extralung sites, the majority of young paragonimus penetrate in the diaphragma and get to the pleural cavity and to lung parenchyma, where the young paragonimus may cause bronchopneumonia, interstitial pneumonia and bronchiectasis [4]. Around the parasite, emerges a group of eosinophils and neutrophils, and later on mononuclear cells. Necrosis of pulmonary parenchyma followed by the development of a fiber capsule surrounding the adult paragonimus happens in a next stage. By the seventh or eighth week, the eggs production starts inside the capsule, which size increases and might break often inside the bronchiolus. The expectorated material has parasite eggs, Charcot-Leyden's crystals, inflammatory cells and necrotized tissue.

The presence of eggs on the tissue leads to the development of granulomatous reaction. When the cysts do not have more viable parasites or are empty, they draw back and a scar may take place and become calcified [5]. Pleural effusion with empyema formation may appear with the parasite migration to the pleural cavity [6]. The disease can be spread to other areas of the organism [7].

The diagnosis is given finding parasites eggs in biologic specimens and/or the parasite identification in a histopathologic exam. Molecular biology techniques have been developed [8]. Serologic methods and ELISA have been used with sensibility and specificity of, respectively, 92\% and 90\%; nevertheless, the values remain high after starting the treatment [9]. The treatment of choice is the praziquantel [10]. 


\section{Case Report}

SSP, 59 years old, white female Brazilian patient, married, teacher, based in Salvador, Bahia, northeastern side of Brazil. The irritative low-productive cough started in July 1998 associated with progressive dyspnea. Eighteen months afterwards, the patient was dyspneic even when resting, when she was then hospitalized. It was reported that, within the 2 years before the disease had showed up, the patient traveled twice to overseas - to Chile and Spain - and reported that she had eaten grilled salmon. The patient used to eat paella, a typical Spanish meal, that is made of seafood and crustaceous specimens. The physical exam presented diffuse rales in both hemithoraces. The result of the first leukogram showed 14,400 cells $/ \mu \mathrm{L}$ with $14 \%$ of eosinophils and the chest x-ray showed peribronchovascular infiltrate, predominating in lower half lung fields, and small opaque nodules (Figure 1). The high-resolution computed tomography scan of the chest (HRCT) presented compatible pattern of airways disease, especially from small airways, with air trapping, tree sprouting images, central lobular nodules and bronchiectasis, making the results compatible with bronchiolitis and bronchiectasis (Figure 2). The initial spirometry revealed VC of $75 \%$ of what was expected and normal $\mathrm{FEV}_{1}$. After 18 months, the result of the VC was $42 \%$ of what was expected.

A treatment with corticoid took action and a transbroncho biopsy revealed cartilaginous bronchus and granulomatous lesion with necrosis, where was observed a structure similar to a parasite capsule (Figures 3 and 4). An exam made with the sputum parasites eggs was indicative for paragonimus, and then sustained by "Center for Control Diseases -USA" (Figure 5).

Praziquantel was used in the treatment. After the use, the patient presented thick ferruginous expectoration, where paragonimus eggs were found again and the BAAR had a positive result. Dyspnea became worse, so the patient was hospitalized. After examination, the patient presented regular general state, no fever, cyanosis with respiratory frequency of $46 \mathrm{bpm}$, heart rate of $120 \mathrm{bpm}$, artery pressure of 110 x 80 $\mathrm{mmHg}$ and there were rales in the chest auscultation of both hemithoraces. At this time, the $\mathrm{O}_{2}$ pulse saturation was 84 mmHg. The treatment for M. tuberculosis and MAC complex was initiated, and the treatment with praziquantel was repeated. The PCR exam and sputum culture were positive for $M$. tuberculosis, and the treatment for MAC-complex was then interrupted.

The patient presented a progressive recover. Nowadays the respiratory frequency is $20 \mathrm{bpm}$ and the pulse saturation is 94\% when resting, decreasing to $91 \%$ after a 6 minutes walk. The last spirometry revealed VC of $52 \%$ of what was expected and the chest HRCT presented subtotal retrocession of the lesions, but it was also noticed the remaining of shortness of breath, observed during expiration (Figures 6 and 7).

\section{Discussion}

It is related to be the first case of paragonimiasis in Brazil. In South America, the majority of paragonimiasis reports had been in Ecuador, with an annual incidence of 13.2 cases [11]. The clinic manifestations of cough, ferruginous sputum (result of the cyst rupture) and discovery of nodules and bronchiectasis on the x-ray examination are the most frequent features [4], and all have been noticed on this current case. The association with $M$. tuberculosis, in endemic area, is described in up to 30\%. More than half of the cases are initially treated as tuberculosis [5,12]. Bronchiectasis and small multiple nodules had been described in the chest HRCT scanning, with positive culture for MAC complex [13]. These notes justify the decision of, initially, treat for both mycobacteria. The accomplishment of PCR for MAC complex and $M$.

Figure 1. X-ray showed peribronchovascular infiltrate, predominating in lower half lung fields, and small opaque nodules.

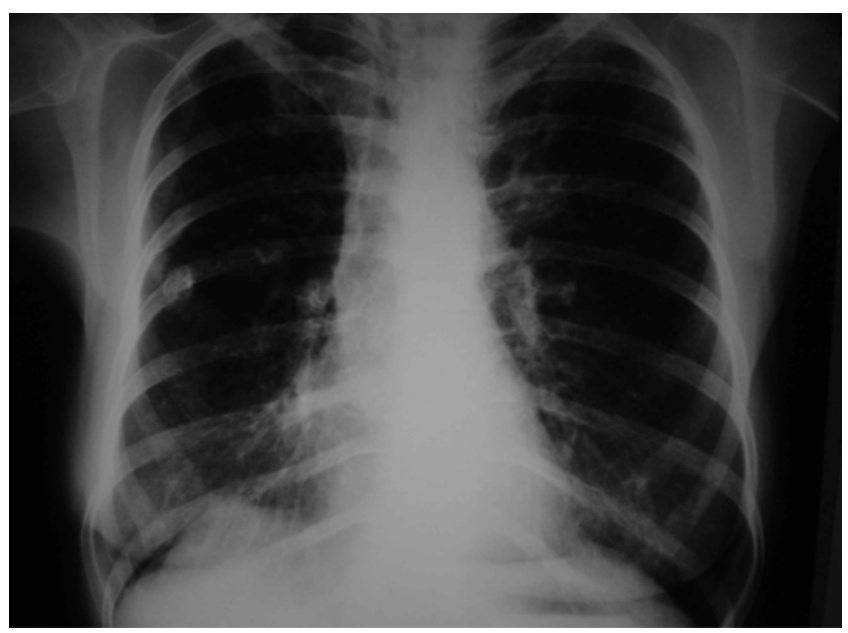

Figure 2. The high-resolution computed tomography scan of the chest (HRCT) presented air trapping, tree sprouting images, central lobular nodules and bronchiectasis, indicating bronchiolitis and bronchiectasis.

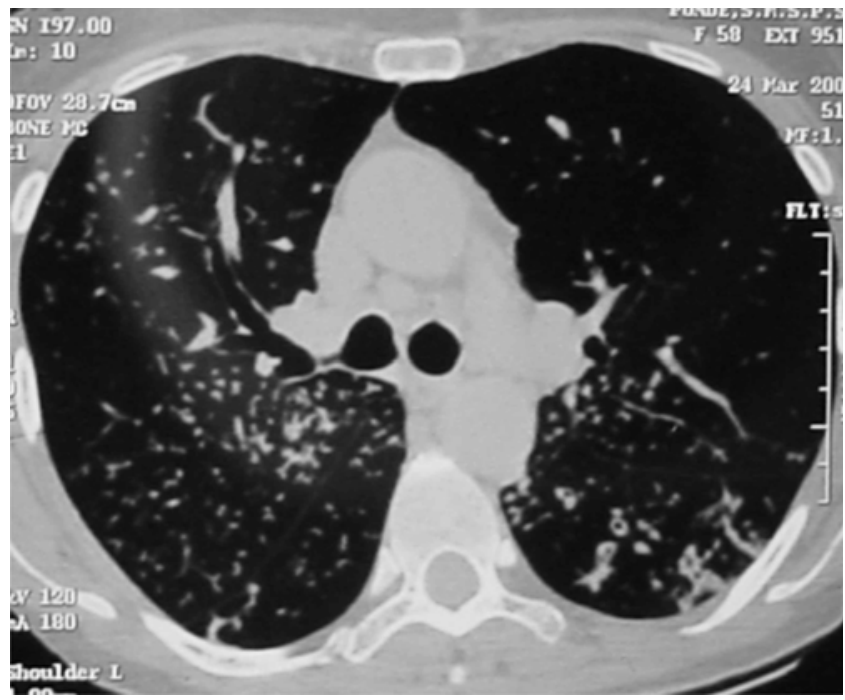


Figures 3 and 4. Transbroncho biopsy revealed cartilaginous bronchus and granulomatous lesion with necrosis.

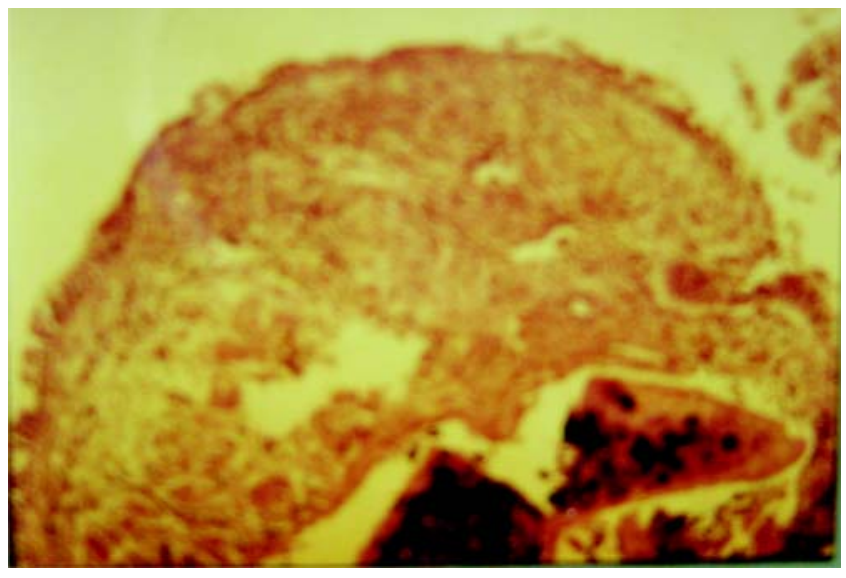

Figure 5. Parasite egg (paragonimus).

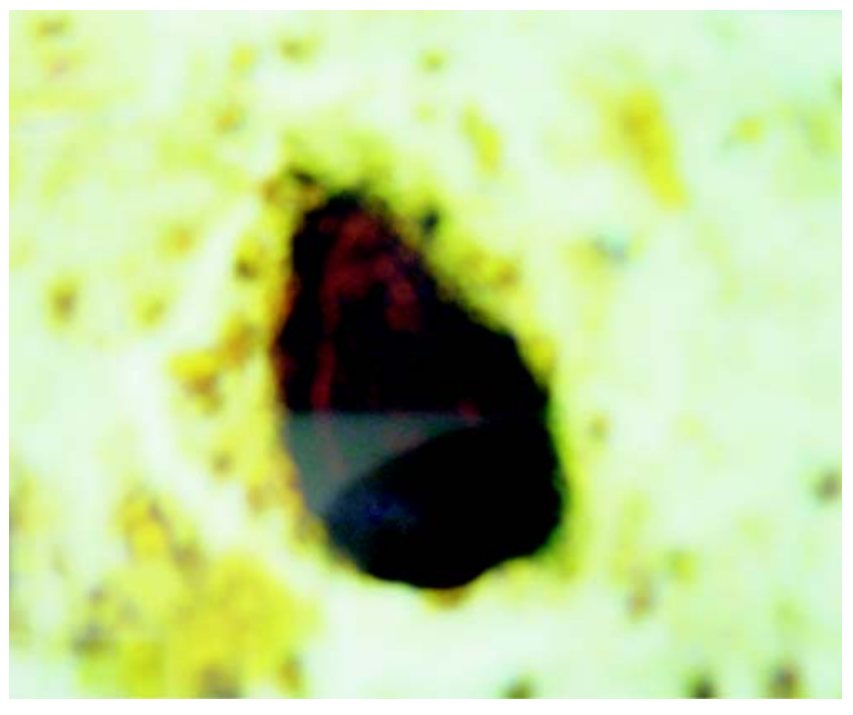

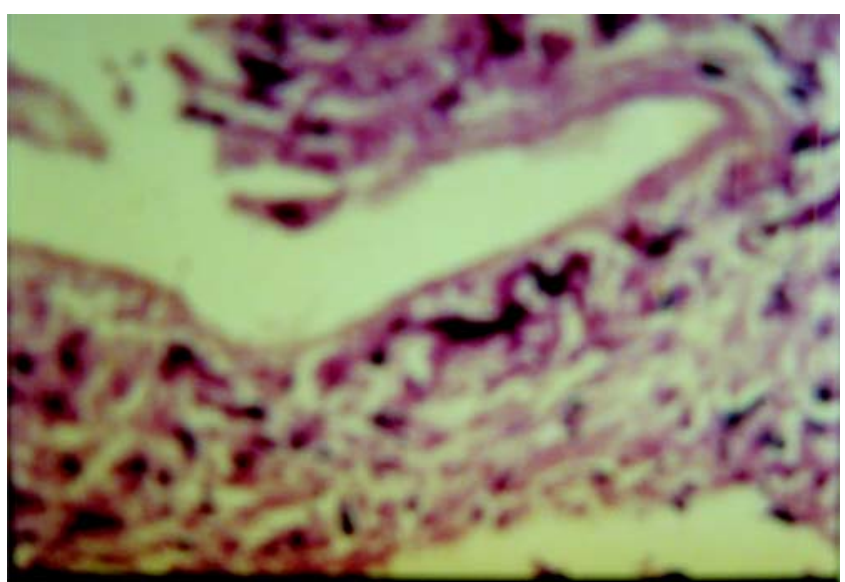

tuberculosis, should be indicated in this situation because it is a very fast exam.

However, the discovery of granulomatous bronchiolitis with caseous necrosis leading to respiratory insufficiency is not a very common finding caused by $M$. tuberculosis infection. Lemos et al related a case of granulomatous bronchiolitis with caseous necrosis due to $M$. tuberculosis that developed with breathing insufficiency, resulting in death, despite the treatment with tuberculostatic drugs [14]. On the authors' view, bronchiolitis on the current case was caused by paragonimus, and this was confirmed by transbroncho biopsy. The shortness of breath observed on the chest HRCT and the reduction of $\mathrm{VC}$ are more likely to be a consequence of a constrictive bronchiolitis, diffuse and residual, than a result of a peribronchiolar scar process after treatment.

Due to the fact that no cases of autochthonous paragonimiasis had been reported in Brazil, the authors concluded that the contamination could had happened as a consequence of the economics globalization process, where

Figures 6 and 7. Chest HRCT presented subtotal retrocession of the lesions. Remaining of shortness of breath, observed during expiration (Figure 7).
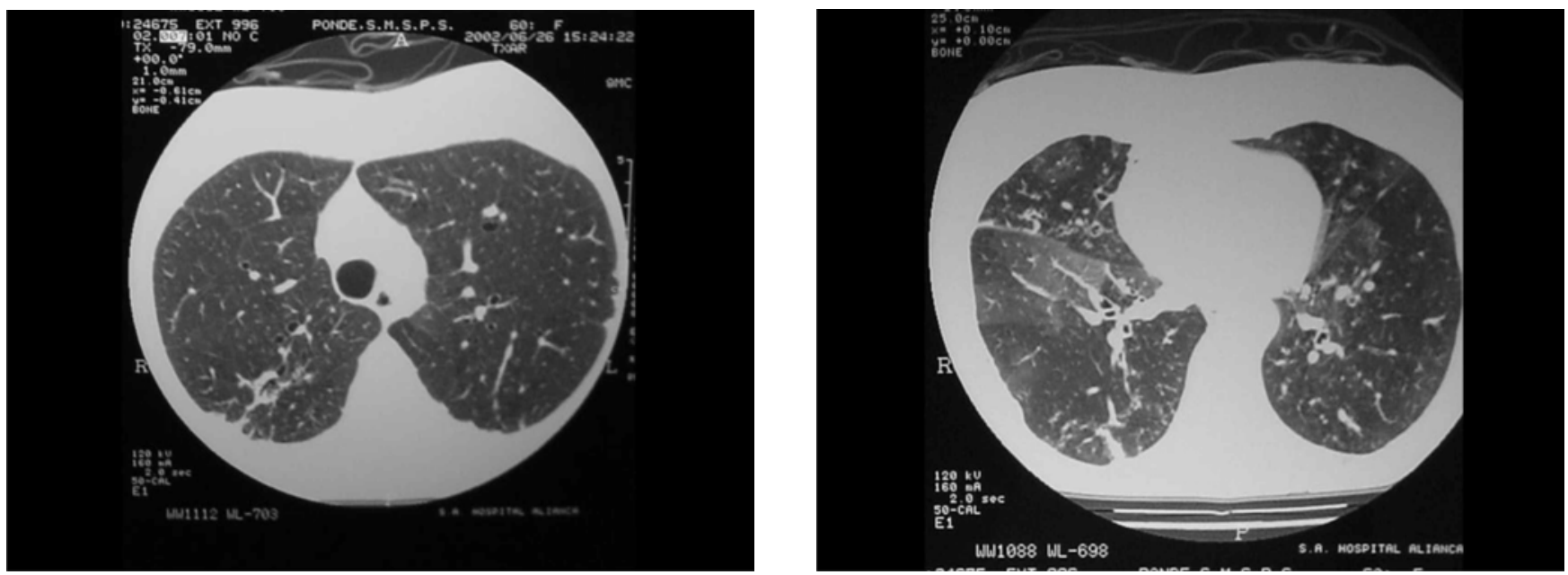
importation of parasitized crustaceans may be the cause. However, there is still necessity to evaluate this theory and even to verify if the coastal region of Bahia, northeastern side of Brazil, area with plenty of rivers and mangroves, where there are a large quantity of mollusks and crustaceans, and also where the patient always had lived, has or not paragonimus specimens.

\section{References}

1. Choi D. Paragonimus and paragonimiasis in Korea. Kisaengchunghak Chapchi 1990;28:79-102.

2. Harinasuta T., Pungpak S., Keystone J.S. Trematode infections. Opisthorchiasis, clonorchiasis, fasciolasis and paragonimiasis. Infect Dis Clin North Am 1993;7:699.

3. Pachucki C., Levandowski R.A., Brown V.A. American paragonimiasis treated with praziquantel. New Engl J Med 1984;311:582.

4. Kagawa F. Pulmonary paragonimiasis. Semin Respir Infect 1997;12:149-58.

5. Nana A., Bovornkitti S. Pleuropulmonar paragonimiasis. Semin Respir Med 1991;12:46-54.
6. Johnson R. Paragonimiasis in Indochinese refugees. Am Rew Respir Dis 1980;128:534-8.

7. Kusner D., King C.H. Cerebral paragonimiasis. Semin Neurol 1993;13:201.

8. Maleewong W. Recent advances in diagnosis of paragonimiasis. Southeast Asian J Trop Med Public Health 1997;28:134.

9. Imai J. Evaluation of ELISA for the diagnosis of paragonimiasis westermani. Trans R Soc Trop Med Hyg 1987;81:3.

10. Johnson J., Jong E.C., Dunning S.B., et al. Paragonimiasis: diagnosis and the use of praziquantel in treatment. Rev Infect Dis 1985; 7:200.

11. Calvopina H., Aguirre L.F., Falcone M.C., et al. Paragonimiasis pulmonar en lo Hosspital de el Carmen, Manbí, Ecuador. Educ med contin 1994;44:17-21.

12. Johnson J., Iber C. Paragonimiasis in United States. A report of nine cases in the Hmong immigrants. Chest 1982;82:168-71.

13. Swensen J., Hartman T.E., Wlliams D.E. Computed tomographic diagnosis of Mycobacterium avium-intracellulare complex in patients with bronchiectasis. Chest 1994;105:49-52.

14. Lemos A., Guanaes A.G. Bronquiolite granulomatosa devido a Micobacterium tuberculosis. Jornal de Pneumologia 1990. 